\title{
Effects of pushing height on trunk posture and trunk muscle activity when a cart suddenly starts or stops moving
}

\author{
Yun-Ju Lee ${ }^{\mathrm{a}}$, Marco J.M. Hoozemans ${ }^{\mathrm{a}}$ and Jaap H. van Dieën ${ }^{\mathrm{a}, *}$ \\ ${ }^{a}$ Research Institute MOVE, Faculty of Human Movement Sciences, VU University Amsterdam, Van der \\ Boechorststraat 9, NL-1081 BT, Amsterdam, The Netherlands
}

\begin{abstract}
Unexpected sudden (un)loading of the trunk may induce inadequate responses of trunk muscles and uncontrolled trunk motion. These unexpected perturbations may occur in pushing tasks, when the cart suddenly starts moving (unloading) or is blocked by an obstacle (loading). In pushing, handle height affects the user's working posture, which may influence trunk muscle activity and trunk movement in response to the perturbation. Eleven healthy male subjects pushed a $200 \mathrm{~kg}$ cart with handles at shoulder and hip height in a start condition (sudden release of brakes) and a stop condition (bumping into an obstacle). Before the perturbation, the baseline of the trunk inclination, internal moment and trunk extensor muscle activity were significantly higher when pushing at hip height than at shoulder height. After the perturbation, the changes in trunk inclination and internal moment were significantly larger when pushing at shoulder height than at hip height in both conditions. The opposite directions of changes in trunk inclination and internal moment suggest that the unexpected perturbations caused uncontrolled trunk motion. Pushing at shoulder height may impose a high risk of low-back injury due to the low trunk stiffness and large involuntary trunk motion occurring after carts suddenly move or stop.
\end{abstract}

Keywords: manual material handling, occupational biomechanics, trunk muscle control, unexpected perturbation

\section{Introduction}

Pushing had been associated with the risk of lowback pain in spite of low mechanical loading of the back $[9,10,19]$. The low moments around the lumbar spine in pushing, probably coincide with a relative low trunk stiffness [3], which may put the spine at the risk of mechanical injury when trunk perturbations occur [4], in particular when transporting objects with high inertia $[2,18]$.

Both sudden loading and unloading perturbations of the trunk may induce inadequate responses of trunk muscles and uncontrolled trunk motion, which are considered potential causes of low-back injury $[5,6]$. This kind of trunk perturbations could occur in pushing tasks. In the initial phase of pushing tasks, the transition from static to dynamic friction causes a sudden drop in hand force as the cart starts moving, which can be considered as a sudden unloading perturbation. When a cart suddenly stops moving, for example due to an obstacle, the increase in hand reaction force can be considered a sudden loading perturbation.

The load at the low-back in pushing is determined by the external force at the hands in combination with the posture and movements of the upper body [12]. Handle height is considered a crucial ergonomic factor $[13,16]$, which influences pushing capability [21], trunk inclination and the magnitude and direction of hand forces $[1,7,11]$. The differences in trunk loading between handle heights that will result may affect trunk muscle control in response to perturbations in cart pushing.

Therefore, the objective of the present study was to investigate how handle height affects trunk inclination and trunk muscle activity in dealing with the

\footnotetext{
* Corresponding author. Tel.: +31 2059 88501; fax: +3120 4448529. E-mail addresses: j.vandieen@fbw.vu.nl (Jaap H. van Dieën).
} 
perturbations occurring when a cart unexpectedly starts and stops moving. We hypothesized that trunk motion would be more affected when pushing at shoulder height than at hip height. As higher trunk moments and hence higher muscle activity would be present prior to the perturbation when pushing at hip height [11]. Additionally, we hypothesized that both types of perturbations (sudden starts and stops) could lead to uncontrolled trunk motion, i.e. an increase in trunk inclination opposite in direction to the change in internal trunk moment. Furthermore, when experiencing unexpected continuous perturbations during pushing while walking [15] and in lifting [23], subjects respond by stiffening the trunk using cocontraction. Therefore, we hypothesized that trunk flexor and extensor would co-contract in response to perturbations in both types of sudden cart movements.

\section{Methods}

\subsection{Subjects}

Eleven healthy male volunteers (age 29.5(SD 5.0) years, height 1.86 (SD 0.06) $\mathrm{m}$ and weight (79.7 (SD $8.4) \mathrm{kg}$ ) participated in the experiment after signing an informed consent. Subjects reported no history of low-back pain or other musculoskeletal disorders within the past 12 months. The ethics committee of the Faculty of Human Movement Science approved the experiment.

\subsection{Experimental design}

A $200 \mathrm{~kg}$ four-wheeled cart (height $1.6 \mathrm{~m}$, depth $0.8 \mathrm{~m}$, width $0.64 \mathrm{~m})$ with hard rubber wheels $(0.028$ $\mathrm{m}$ wide, diameter $0.124 \mathrm{~m}$ ) was used. The two wheels nearest to the subjects could swivel. Force transducers were attached to the two handles, at the subject's shoulder height (acromion angle) or hip height (upper border of greater trochanter). Two remote-controlled caliper brakes attached to the front wheels could be used to prevent the cart from moving (Figure 1). An obstacle, a metal bar (length $63 \mathrm{~cm}$, height $2.8 \mathrm{~cm}$. width $7 \mathrm{~cm}$ ), was attached to the cart just in front of the front wheels and could be released by an electromagnet controller.

\subsection{Procedure}

Prior to start the experimental pushing activities, subjects performed a series of contractions meant to elicit the maximum isometric voluntary contractions
(MVC) of each of the trunk muscles studied [17]. Then, subjects familiarized themselves with the task of pushing a cart for about $5 \mathrm{~min}$. In order to create an unexpected perturbation, a random number of $(5$ or 6) reference trials was performed to the perturbed (start and stop) conditions. For the start condition, the reference trial was the brakes were not released, in the following trial the brakes were suddenly and unexpectedly released. For the stop condition, the reference trail was pushing the cart while walking over a 5 $\mathrm{m}$ distance at a self-selected speed, in the following trial the obstacle was suddenly released at mid-stance of the right foot. The sequence of the tasks, i.e. two perturbation conditions (start and stop) at two handle heights (shoulder and hip heights), was randomized.



Fig. 1: The experimental setup, showing the four-wheeled cart instrumented with two caliper brakes on the front wheels and an electromagnetic device holding an obstacle in front of the front wheels.

\subsection{Data acquisition}

Hand forces and kinematic data of LED cluster markers on the upper body segments were collected by $3 \mathrm{D}$ force transducers (SRMC3A series, Advanced Mechanical Technology, Inc., USA) and an Optotrak system (Northern Digital, Waterloo ON, Canada), respectively. Force data were stored at 1000 sam$\mathrm{ples} / \mathrm{s}$ and then reduced to $50 \mathrm{samples} / \mathrm{s}$ using a running average. Clusters of three LED markers were attached to a $50 \mathrm{~mm}$ equilateral triangle metal plate on a double hinge joint. Clusters were placed on the pelvis, thorax, bilateral upper arms and forearms and additional markers were placed at the handles of the 
cart and feet. Marker positions were recorded at 50 samples/s. The internal moment at the L5-S1 intervertebral disc was estimated from the reaction forces at the hands and the anthropometry and kinematics of upper body segments, using an inverse dynamic model [14]. Markers on the handles were used to calculate the position of the cart and the onset of cart movement in the start condition. Markers on the feet were used to monitor the gait pattern on-line during the trials, to detect mid-stance of the right foot for obstacle release.

Electromyograms (EMG) were recorded by using disposable $\mathrm{Ag} / \mathrm{AgCl}$ surface-electrodes (Blue Sensor; lead-off area $1.0 \mathrm{~cm}^{2}$, inter-electrode distance $2.5 \mathrm{~cm}$ ). After abrasion and cleaning with alcohol, electrodes were bilaterally attached over internal oblique (OI: 3 $\mathrm{cm}$ medial to the anterior superior iliac spine (ASIS)), external oblique (OE: halfway the axial line between the $10^{\text {th }}$ rib and the ASIS), rectus abdominis (RA: 3 $\mathrm{cm}$ lateral to the umbilicus), multifidus (MU: $2 \mathrm{~cm}$ lateral to L4-L5), longissimus thoracis pars lumborum (LL: $3 \mathrm{~cm}$ lateral to L3), iliocostalis lumborum (IL: $6 \mathrm{~cm}$ lateral to L2), iliocostalis thoracis (IT: $6 \mathrm{~cm}$ lateral to T11) and longissimus thoracis pars thoracis (LT: $3 \mathrm{~cm}$ lateral to T10). EMG signals were band-pass filtered $(10-400 \mathrm{~Hz})$, amplified (20 times, Porti-17TM, TMS, Enschede, The Netherlands; input impedance $>10^{12} \Omega$, common mode rejection ratio $>90 \mathrm{~dB}$ ) and stored on disk (sample rate 1000 samples/s; 22 bits). ECG contamination was identified by means of independent component analysis and removed from the signals [15]. Subsequently, EMG signals were high-pass filtered at $20 \mathrm{~Hz}$ and band-stop filtered at $50 \mathrm{~Hz}$ and finally full-wave rectified and low-pass filtered at $2 \mathrm{~Hz}$ (2nd order Butterworth). The signals of a series of MVC trials were processed using the same steps and the maximal values were used to normalize the EMG signals.

\subsection{Data analysis}

\subsubsection{For the start condition}

After normalization to the MVC values, EMG amplitudes of bilateral RA, OE and OI were averaged to represent trunk flexor muscle activity and bilateral MU, LL, IL, IT and LT EMG amplitudes were averaged to represent trunk extensor muscle activity. The average values of the trunk inclination and the internal moment in the sagittal plane, trunk flexor muscle and extensor muscle activity of the second before cart movement were considered as the baseline values.
To determine changes after the onset of cart movement, the peak values of trunk inclination, internal moments, and trunk flexor and extensor muscle activity observed in the first second after movement of the cart were determined. Subsequently, the difference between the peak values after cart movement and the baseline values were considered as the maximum change.

\subsubsection{For the stop condition}

Trunk inclination and the internal moment at L5S1 in the sagittal plane and trunk flexor and extensor muscle activity were analyzed for the first second after the obstacle was released and after the midstance phase of the right foot after walking over a 2.5 $\mathrm{m}$ distance in the reference trial. Five random reference trials were averaged to represent the reference condition. The peak values of trunk inclination, internal moments, and trunk flexor and extensor muscle activity during the one-second time series were determined. In the reference condition, the range from mean plus to minus one standard deviation was considered as the normal range of pushing while walking. Subsequently, the difference between the peak in the stop condition and the edge of the normal range at the same instant in the one-second time series was considered as the maximum change due to the perturbation. In addition, the mean value at the same instant was defined as the baseline.

\subsection{Statistics}

Data were checked for normality before analysis. As most of the data appeared to be skewed to the right, data were logarithmically transformed. As mentioned above, the start and stop conditions are in the initial and end phases of the pushing tasks, in which the reaction forces are in the opposite direction. Hence, separate paired-sample t tests with handle height as the independent variable were used for the start and stop conditions, separately. For the start condition, the baseline values and maximum changes of trunk inclination, internal moment, and trunk flexor and extensor muscle activity were compared between pushing at shoulder height and hip height. For the stop condition, the baseline and maximum changes of the same dependent variables were compared between pushing at shoulder height and hip height. For all tests, $p$ values $<0.05$ were considered statistically significant. 


\section{Results}

\subsection{For the start condition}

A typical example of the data in the start condition of one subject is shown in the left panels of Figure 2. The vertical lines represent the instant of cart movement after the sudden release of the brakes and the data are presented for $200 \mathrm{~ms}$ before this instant and $1 \mathrm{~s}$ after this instant. Before the cart started to move, trunk inclination, internal moment and trunk flexor and extensor muscle activity were nearly constant but different between pushing at shoulder height and hip height. At the group level, handle height significantly affected trunk inclination, internal moment and trunk extensor muscle activity (Table 1 ). When pushing at hip height, higher trunk extensor muscle activity (8.42 SD 4.32\%MVC) coincided with higher trunk inclination $\left(26.84 \mathrm{SD} 13.89^{\circ}\right)$ compared to pushing at shoulder height $(2.76$ SD $2.30 \% \mathrm{MVC} \& 14.09$ SD $5.93^{\circ}$ ), as shown in the right panels of Figure 2 .
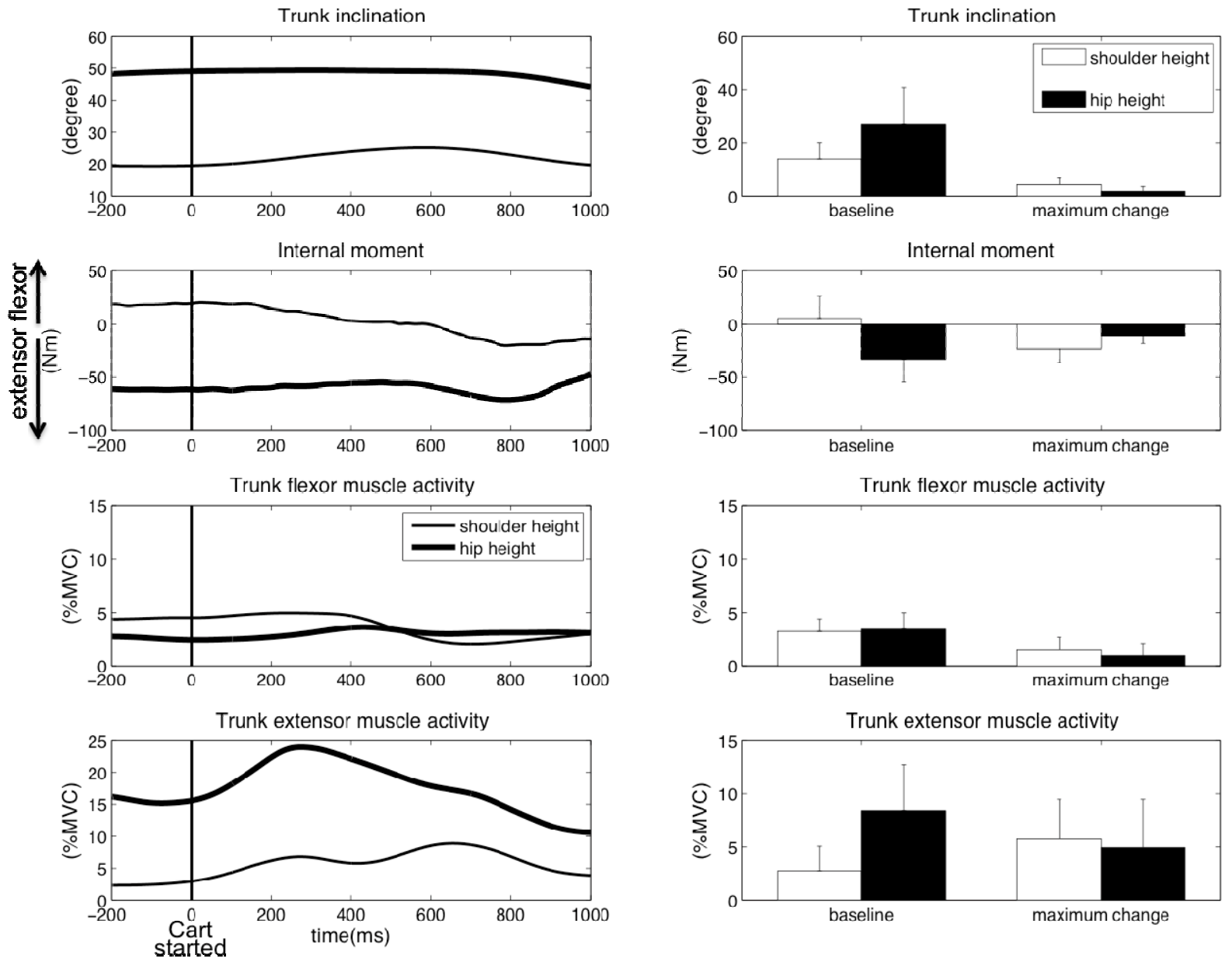

Fig. 2: Typical example of data from pushing at shoulder height and hip height in the start condition (left panels). The vertical lines represent the onset time of cart starting moving. Positive and negative internal moments represent flexor and extensor moments, respectively. Positive trunk inclination represents flexion. Mean and standard deviations (error bars; left panels). The right and left clusters of bars represent baseline values and maximum changes, respectively. 
When pushing at hip height, trunk inclination and internal moment remained nearly constant after the cart started to move. In contrast, when pushing at shoulder height, an increase in trunk inclination coincided with a decrease in internal moment (i.e. a decrease in flexor moment followed by an increase in extensor moment). The latter suggests that the trunk motion was not induced by the internal moment, but by the external moment due to gravity only to be slowed down by the internal moment. Both trunk flexor and extensor muscle activity increased in response to the cart movement in pushing at shoulder and hip height. In line with our hypothesis, at the group level (Table 1), maximum changes in trunk inclination and internal moment were significantly larger when pushing at shoulder height (4.36 SD $\left.2.48^{\circ} \&-23.10 \mathrm{SD} 12.91 \mathrm{Nm}\right)$ than hip height $(1.84$ SD $1.85^{\circ} \&-11.58$ SD $6.86 \mathrm{Nm}$ ). However, handle height did not affect trunk muscle activity after the perturbation and the magnitudes of maximum change are similar at both handle heights.
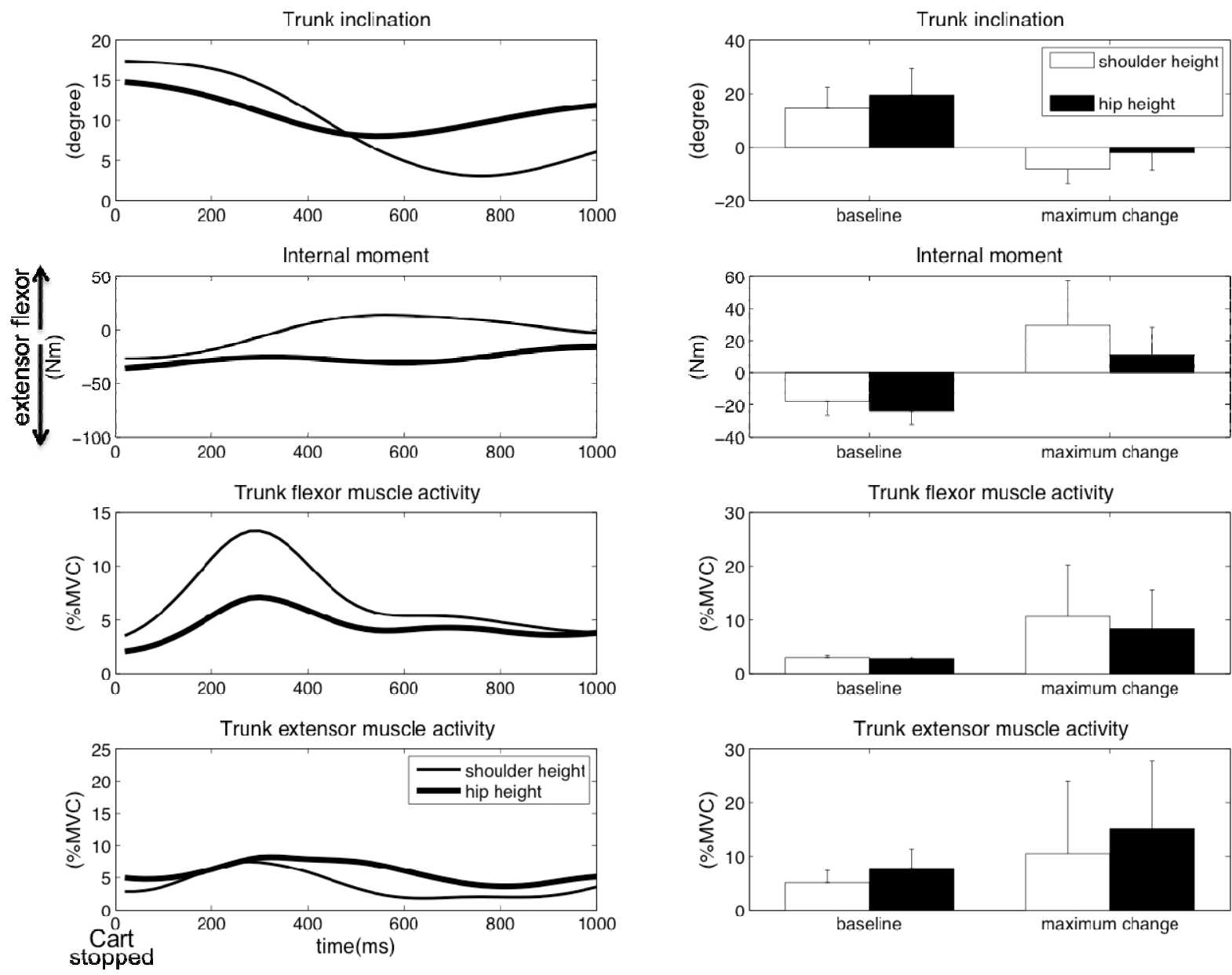

Fig. 3: Typical example of data in pushing at shoulder height and hip height in the stop condition (left panels). The $0 \mathrm{~ms}$ represent the time that the cart was blocked. Positive and negative internal moments represent flexor and extensor moments, respectively. Positive trunk inclination represents flexion. Mean and standard deviations (error bars; left panels). The right and left clusters of bars represent baseline values and maximum changes, respectively. 
Table 1

Results of the paired-samples $t$ test to determine the differences between shoulder height and hip height

\begin{tabular}{llccccccc}
\hline $\begin{array}{l}\text { Paired-samples } t \text { test } \\
\text { (shoulder height vs. hip height) }\end{array}$ & \multicolumn{1}{l}{ Start } & \multicolumn{3}{l}{ Stop } \\
\cline { 2 - 8 } & Baseline & \multicolumn{3}{c}{ Maximum change } & Baseline & \multicolumn{2}{c}{ Maximum change } \\
\cline { 2 - 8 } & $t(10)$ & $p$ & $t(10)$ & $p$ & $t(10)$ & $p$ & $t(10)$ & $p$ \\
\hline Trunk inclination & -3.213 & $\mathbf{0 . 0 0 9}$ & 3.240 & $\mathbf{0 . 0 0 9}$ & -1.926 & 0.083 & -2.890 & $\mathbf{0 . 0 1 6}$ \\
Internal moment & 3.987 & $\mathbf{0 . 0 0 3}$ & -2.263 & $\mathbf{0 . 0 4 7}$ & 2.928 & $\mathbf{0 . 0 1 5}$ & 2.279 & $\mathbf{0 . 0 4 6}$ \\
Trunk flexor muscle activity & -0.336 & 0.744 & 1.123 & 0.288 & 0.997 & 0.342 & 0.678 & 0.513 \\
Trunk extensor muscle activity & -4.661 & $\mathbf{0 . 0 0 1}$ & 1.113 & 0.292 & -6.514 & $<\mathbf{0 . 0 0 1}$ & -2.492 & $\mathbf{0 . 0 3 2}$ \\
\hline
\end{tabular}

\subsection{For the stop condition}

A typical example of the data in the stop condition of one subject is shown in the left panels of Figure 3. The $0 \mathrm{~ms}$ is the instant the cart stopped at mid-stance of the right foot and data are presented for 1 second after this instant. A decrease in trunk inclination coincided with an increase in trunk flexor moment when pushing at shoulder height, similar to the start condition, but in the opposite direction. The same patterns with smaller changes in trunk inclination and internal moment were observed when pushing at hip height. Furthermore, increases in trunk flexor and extensor muscle activity occurred, both when pushing at shoulder and hip height, again similar to the patterns of muscle activity in the start condition.

The baselines were the mean value at the same instant with the maximum change, which was significantly affected by handle height (Table 1). As shown in right panels of Figure 3, internal moment and EMG amplitude of trunk extensor muscle were significantly higher when pushing at hip height (-23.83 SD $8.56 \mathrm{Nm} \& 7.76 \mathrm{SD} 3.57 \% \mathrm{MVC})$ than at shoulder height (-17.48 SD $9.37 \mathrm{Nm}$ \& $5.06 \quad$ SD $2.31 \% \mathrm{MVC})$. This was similar with the start condition in which before the perturbation pushing at hip height provides higher internal moment and trunk extensor muscle activity compared to pushing at shoulder height. As expected, handle height significantly affected maximum changes in trunk inclination, internal moment and EMG amplitudes of trunk muscles, except trunk flexors (Table 1). At group level (right panels of Figure 3), maximum changes in trunk inclination $\left(-8.16 \mathrm{SD} 5.27^{\circ}\right)$ and internal moment (29.39 SD $27.84 \mathrm{Nm}$ ) were significantly larger when pushing at shoulder height than at hip height $(-2.10$ SD $6.48^{\circ} \& 11.31 \mathrm{SD} 16.86 \mathrm{Nm}$ ). This suggests that the perturbation had less impact on the trunk when pushing at hip height compared to shoulder height. Additionally, the maximum change in trunk extensor muscle activity was 15.04 (SD 12.70) \%MVC when pushing at hip height, which was significantly higher than when pushing at shoulder height (10.50 SD $13.51 \% \mathrm{MVC})$.

\section{Discussion}

The present study was designed to investigate how handle height affects trunk inclination and trunk muscle activity after a cart suddenly starts and stops moving. When pushing at hip height, the displacement of the trunk was smaller after the perturbations compared to pushing at shoulder height. In both conditions, trunk internal moment and trunk motion had opposite directions and trunk flexor and extensor muscles co-contracted in response to the perturbations.

Higher postural activity of back muscles is generally associated with increased trunk inclination [20], which was confirmed by the higher baseline EMG values in pushing at hip height compared to pushing at shoulder height. Higher trunk muscle activity elicited by external loading was previously shown to enhance trunk stability $[3,8,24]$ and to attenuate the effects of external perturbations on trunk posture $[3,22]$. Hence, the trunk stiffness is probably relatively low when pushing at shoulder height compared to hip height, which would imply that the impact of a perturbation is different between pushing at shoulder height and hip height, as was indeed reflected in changes in trunk orientation after the perturbation, i.e. an increase in trunk flexion in the start condition and an increase in trunk extension in the stop condition. In addition, taking the stop condition as example, the trunk extension that occurred coincided with an external extension moment (opposite to and exceeding the internal flexor moment), which suggests that the perturbation directly caused an involuntary trunk motion. Similarly, opposite trunk motions and moments were observed in the start condition. 
In the start condition, the nearly constant trunk inclination, internal moment and trunk muscle activity before the sudden release of the brakes, with all parameters changing only after the release, suggests that subjects did not anticipate this external perturbation. Similarly, in the stop condition, these parameters deviated from those in the reference trials only after the cart bumped into the obstacle. Taken together, the sudden changes in cart movement (start and stop) formed unexpected perturbation of trunk equilibrium. Subsequent increases changes in trunk flexor and extensor muscle activity occurred, in line with our hypothesis that trunk muscle co-contraction is used to dealing with unexpected and unpredictable perturbation $[15,23]$.

The present study involved young healthy male subjects only. Generalization to the other population, such as skilled workers, should be considered with care.

\section{Conclusion}

Handle height in cart pushing, through differences in trunk inclination and differences in postural activity of the trunk muscle affects the responses in trunk muscle activity and trunk motion after sudden starts and stops of cart movement. Involuntary trunk motion in cart pushing suggests that trunk stability could be threatened by such perturbations. Compared to pushing at shoulder height, pushing at hip height provides higher trunk stiffness before the perturbation and reduces the impact of the perturbations. In other words, pushing at shoulder height may involve a risk of low-back injury when unpredictable perturbation occurs.

\section{References}

[1] K. W. Al-Eisawi, et al., "The effect of handle height and cart load on the initial hand forces in cart pushing and pulling," Ergonomics, 1999, pp. 1099-1113.

[2] D. B. Chaffin, et al., "Low-back stresses when learning to use a materials handling device," Ergonomics, 1999, pp. 94110.

[3] J. Chiang and J. R. Potvin, "The in vivo dynamic response of the human spine to rapid lateral bend perturbation: effects of preload and step input magnitude," Spine (Phila Pa 1976), 2001, pp. 1457-64.

[4] J. Cholewicki and S. M. McGill, "Mechanical stability of the in vivo lumbar spine: implications for injury and chronic low back pain," Clin Biomech (Bristol, Avon), 1996, pp. 115 .
[5] J. Cholewicki, et al., "Delayed trunk muscle reflex responses increase the risk of low back injuries," Spine (Phila Pa 1976), 2005, pp. 2614-20.

[6] J. Cholewicki, et al., "Effects of external trunk loads on lumbar spine stability," J Biomech, 2000, pp. 1377-85.

[7] M. P. de Looze, et al., "Force direction and physical load in dynamic pushing and pulling," Ergonomics, 2000, pp. 377390.

[8] M. G. Gardner-Morse and I. A. Stokes, "Trunk stiffness increases with steady-state effort," J Biomech, 2001, pp. 457-63.

[9] M. J. Hoozemans, "Pushing and pulling in relation to musculoskeletal complaints.," Ph.D. Thesis, University of Amsterdam, Amsterdam, 2001.

[10] M. J. Hoozemans, et al., "Mechanical loading of the low back and shoulders during pushing and pulling activities," Ergonomics, 2004, pp. 1-18.

[11] M. J. Hoozemans, et al., "Cart pushing: The effects of magnitude and direction of the exerted push force, and of trunk inclination on low back loading.," International Journal of Industrial Ergonomics, 2007, pp. 832-44.

[12] M. J. Hoozemans, et al., "Pushing and pulling in relation to musculoskeletal disorders: a review of risk factors," Ergonomics, 1998, pp. 757-81.

[13] M.-C. Jung, et al., "Pushing and pulling carts and twowheeled hand trucks," International Journal of Industrial Ergonomics, 2005, pp. 79-89.

[14] I. Kingma, et al., "Segment inertial parameter evaluation in two anthropometric models by application of a dynamic linked segment model," J Biomech, 1996, pp. 693-704

[15] Y. J. Lee, et al., "Oblique abdominal muscle activity in response to external perturbations when pushing a cart," $J$ Biomech, 2010, pp. 1364-72.

[16] K. Mack, et al., "Usability of manual handling aids for transporting materials," Appl Ergon, 1995, pp. 353-64.

[17] S. M. McGill, "Electromyographic activity of the abdominal and low back musculature during the generation of isometric and dynamic axial trunk torque: implications for lumbar mechanics," J Orthop Res, 1991, pp. 91-103.

[18] M. A. Nussbaum, et al., "Motion times, hand forces, and trunk kinematics when using material handling manipulators in short-distance transfers of moderate mass objects," Appl Ergon, 2000, pp. 227-37.

[19] S. Plouvier, et al., "Biomechanical strains and low back disorders: quantifying the effects of the number of years of exposure on various types of pain," Occup Environ Med, 2008, pp. 268-74.

[20] U. Raschke and D. B. Chaffin, "Support for a linear lengthtension relation of the torso extensor muscles: an investigation of the length and velocity EMG-force relationships," J Biomech, 1996, pp. 1597-604.

[21] C. Shoaf, et al., "Comprehensive manual handling limits for lowering, pushing, pulling and carrying activities," Ergonomics, 1997, pp. 1183-200.

[22] I. A. Stokes, et al., "Decrease in trunk muscular response to perturbation with preactivation of lumbar spinal musculature," Spine (Phila Pa 1976), 2000, pp. 1957-64.

[23] J. H. van Dieën, et al., "Evidence for a role of antagonistic cocontraction in controlling trunk stiffness during lifting," $J$ Biomech, vol. 36, pp. 1829-36, Dec 2003.

[24] F. J. Vera-Garcia, et al., "Effects of abdominal stabilization maneuvers on the control of spine motion and stability against sudden trunk perturbations," $J$ Electromyogr Kinesiol, 2007, pp. 556-67. 